\title{
Neuronal dynamics of signal selective motor plan cancellation in the macaque dorsal premotor cortex
}

A R T I C L E I N F O

Article history:

Received 30 March 2020

Reviewed 20 May 2020

Revised 23 July 2020

Accepted 10 September 2020

Published online 19 November 2020

\section{Keywords:}

Dorsal premotor cortex

Selective inhibitory control

Behavioural strategy

Neuronal activity

\begin{abstract}
A B S T R A C T
Primates adopt various strategies to interact with the environment. Yet, no study has examined the effects of behavioural strategies with regard to how movement inhibition is implemented at the neuronal level. We used a modified version of the stop-task by adding an extra signal - termed the Ignore signal - capable of influencing the inhibition of movements only within a specific strategy. We simultaneously recorded multisite neuronal activity from the dorsal premotor (PMd) cortex of macaque monkeys during the task and applied a state-space approach. As a result, we found that movement generation is characterized by neuronal dynamics that evolve between subspaces. When the movement is halted, this evolution is arrested and inverted. Conversely, when the Ignore signal is presented, inversion of the evolution is observed briefly and only when a specific behavioural strategy is adopted. Moreover, neuronal signatures during the inhibitory process were predictive of how PMd processes inhibitory signals, allowing the classification of the resulting behavioural strategy. Our data further corroborate the PMd as a critical node in movement inhibition.
\end{abstract}

(c) 2020 Elsevier Ltd. All rights reserved.

\section{Introduction}

Primates have the crucial ability to suppress actions rapidly, a capacity that can be strategically regulated. For example, they can decide to delay an on-going action if something occurs suddenly in the environment: this momentary pause could allow them to better evaluate the consequence of the action and decide their next move. Alternatively, they can choose to ignore the new signal and continue pursuing the initial goal.

Neuroscience studies have typically examined simple forms of movement inhibition in experimental settings,

\footnotetext{
* Corresponding author.

** Corresponding author

E-mail addresses: pierpaolo.pani@uniroma1.it (P. Pani), stefano.ferraina@uniroma1.it (S. Ferraina). 
using the stop (or countermanding) task (Hanes, Patterson, \& Schall, 1998; Logan \& Cowan, 1984). In this task, the primary instruction is to respond as quickly as possible to a Go signal; in a minority of trials a Stop signal is presented after the Go signal, and subjects are required to refrain from moving. The ability to countermand the response is evaluated, based on the estimate of the stop signal reaction time (SSRT). The SSRT can be broadly considered to be the response time of movement inhibition (Logan \& Cowan, 1984) and as such has been used to compare the efficiency of inhibitory control in various populations of patients; during the many stages of brain maturation, from childhood to senescence; and across animal species (Brunamonti et al., 2011; Brunamonti, Ferraina, \& Paré, 2012; Hanes et al., 1998; Hippolyte, Iglesias, \& Barisnikov, 2009; Lijffijt, Kenemans, Verbaten, \& van Engeland, 2005; Lipszyc \& Schachar, 2010; Pani et al., 2013; Pani et al., 2014; Paré \& Hanes, 2003; Williams, Ponesse, Schachar, Logan, \& Tannock, 1999). However, because inhibition can intervene to regulate behaviour in many ways, in recent years, more complex tasks have been used to determine the behavioural consequences of and the neuronal functional architecture that underlies complex inhibitory control (Aron, 2010; Bissett \& Logan, 2014; Boehler, Appelbaum, Krebs, Chen, \& Woldorff, 2011; Cai, Oldenkamp, \& Aron, 2011; Chikazoe et al., 2009; Majid, Cai, George, Verbruggen, \& Aron, 2012; Sebastian et al., 2016, 2017; Sharp et al., 2010; Xu et al., 2017; Xu, Mayse, \& Courtney, 2018). Among the tasks that have been proposed, the selective stop task (Bissett \& Logan, 2014) entails the presentation of a Stop signal or an Ignore signal after the Go signal in a subset of trials, of which only the Stop signal requires inhibition of the movement.

Behavioural studies in humans (Bissett \& Logan, 2014; Sánchez-Carmona, Albert, \& Hinojosa, 2016; Sebastian et al., 2017) demonstrated that subjects are able to deal with the task successfully, suppressing the action only when required. Bissett and Logan (2014), first suggested that some subjects adopt the strategy to activate the inhibitory control momentarily procrastinating the discrimination between proposed signals while other subjects adopt the strategy to first discriminate then respond/stop as necessary. Conclusions were based on behavioural evidence only.

In the present study we evaluated the presence of a neuronal correlate of the strategy adopted in monkeys trained in the selective stop task.

Among the different areas involved in the inhibitory control of planned actions, both at the cortical and subcortical levels (Cai et al., 2011; Aron et al., 2007; Wessel \& Aron, 2017; Aron \& Poldrack, 2006; Aron, Fletcher, Bullmore, Sahakian, \& Robbins, 2003; Wagner, Wessel, Ghahremani, \& Aron, 2018; Duque, Labruna, Verset, Olivier, \& Ivry, 2012; Duque, Greenhouse, Labruna, \& Ivry, 2017; Parmigiani \& Cattaneo, 2018; Hanes et al., 1998, Pare'\& Hanes, 2003; Schmidt, Leventhal, Mallet, Chen, \& Berke, 2013; Mallet et al., 2016), we focused on the dorsal premotor cortex (PMd), an area crucial in establishing the transition from decision to action for arm movements; neuronal activity in PMd continuously reflects the accumulation and change in information that is pertinent to the momentary decision state regarding forthcoming movements (Kaufman, Churchland, Ryu, \& Shenoy, 2014; Thura \& Cisek, 2014), and predicts when and whether an arm movement will be generated or inhibited (Kaufman et al., 2016; Mirabella, Pani, \& Ferraina, 2011; Pani et al., 2014, 2018).

By analysing the multisite dynamics of neuronal activity in the PMd, we obtained evidence of a strategy-dependent inhibitory neuronal process, triggered by the Ignore signal only when monkeys adopted a specific strategy. These novel data corroborate the model proposed by Bissett and Logan (2014) in humans and demonstrate, once again, the strong correlation between modulation of the neuronal activity in the PMd and the adopted behaviour, suggesting PMd as a key structure in the cognitive control of movements.

\section{Material and methods}

\subsection{Subjects}

Two male rhesus macaque monkeys (Macaca mulatta; designated Monkeys 1 and 2), weighing 9 and $13 \mathrm{~kg}$, were studied. We designed the study assuming sample sizes similar to those used in other monkey studies (see for example, Genovesio, Brunamonti, Giusti, \& Ferraina, 2007; Pani et al., 2018). The monkeys were pair-housed with cage enrichment. They were fed daily with standard primate chow, supplemented with nuts and fresh fruits. The monkeys received part of their daily water supply during the experiments in the form of fruit juice. All experimental procedures, animal care, housing, and surgical procedures conformed to European (Directive 2010/63/ UE) and Italian (D.L. 26/2014) laws on the use of nonhuman primates in scientific research and were approved by the Italian Ministry of Health.

\subsection{Animal preparation}

At the end of training period (see Supplementary Materials for details about how we trained monkeys in this task) a Utah array (96 channels, Blackrock Microsystems, USA) was surgically inserted in the PMd of each monkey, using the arcuate sulcus (AS) and pre-central dimple (pCD) as anatomical landmarks after opening of the dura (Fig. 1 in Supplementary materials). The recording site was contralateral to the arm used during the experiment. A headholding device was implanted in both monkeys. All surgeries were performed under sterile conditions and veterinary supervision. Antibiotics and analgesics were administered postoperatively. Anaesthesia was induced with ketamine (Imalgene, $10 \mathrm{mg} \mathrm{kg}^{-1}$ i.m.) and medetomidine hydrochloride (Domitor, $.04 \mathrm{mg} \mathrm{kg}^{-1}$ i.m.) and maintained with inhalant isoflurane $(.5 \%-4 \%)$ in oxygen. Antibiotics were administered prophylactically during the surgery and postoperatively for at least 1 week. Postoperative analgesics were given at least twice daily. Recordings of neuronal activity started after a minimum of 10 weeks after recovery from the surgery. 


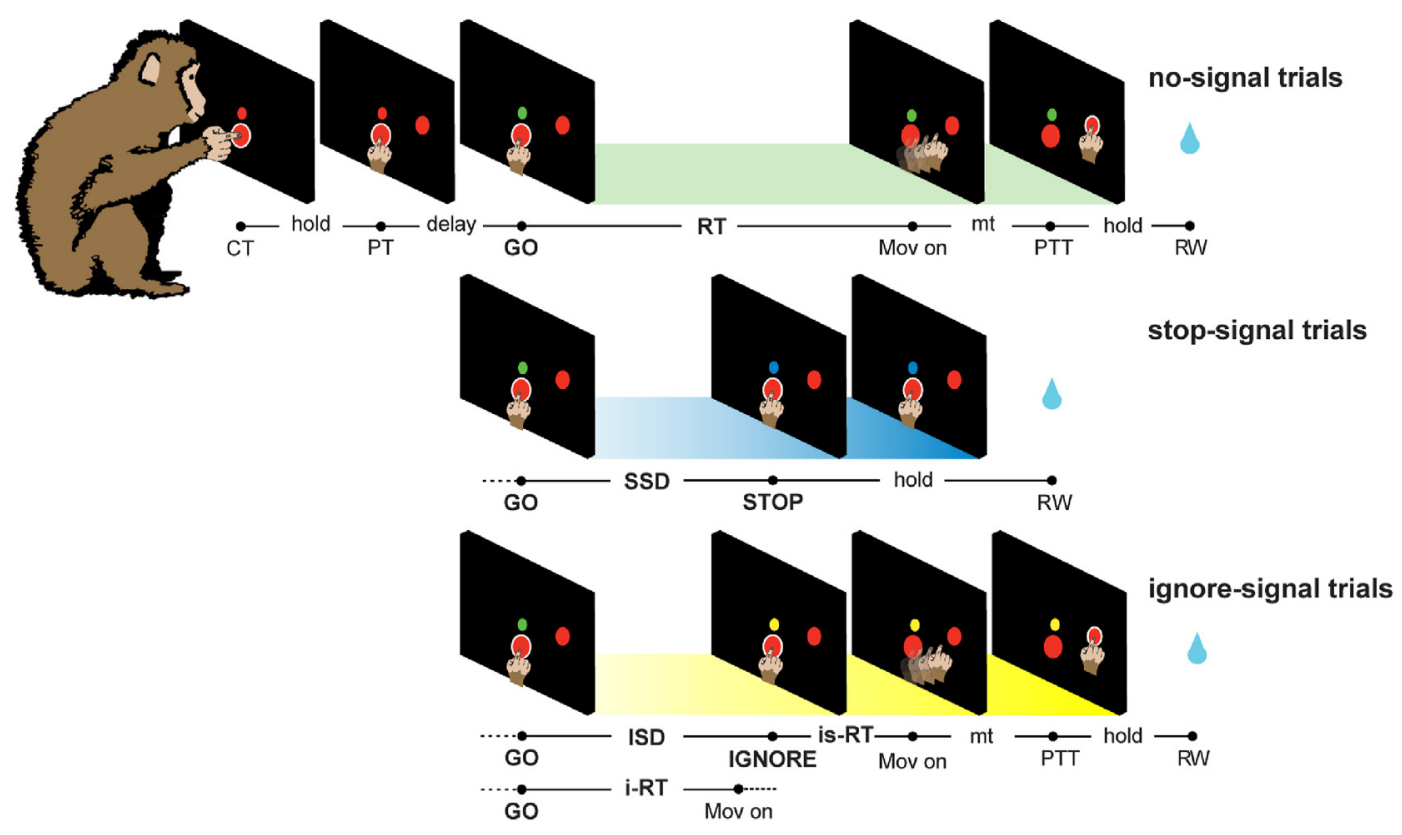

Fig. 1 - Signal selective stop task. Three types of trials were presented (intermingled) in each block. Movement cancellation was required in stop-signal trials only. CT, central target; PT, peripheral target; GO, Go signal; Mov on, movement onset; RT, reaction time; is-RT, Ignore-signal reaction time; i-RT, ignore reaction time; $\mathrm{mt}$, movement time; PTT, peripheral target touch; RW, reward. SSD, stop signal delay; ISD, ignore signal delay; hold, central, or peripheral holding time.

\subsection{Apparatus and task}

Experiments were performed in a darkened, acoustically insulated room. Monkeys were seated in front of a black isoluminant background $\left(<.1 \mathrm{~cd} / \mathrm{m}^{2}\right)$ of a 17 -inch touchscreen monitor (LCD, $800 \times 600$ resolution). A non-commercial software package, CORTEX (www.nimh.nih.gov), was used to control the presentation of stimuli and behavioural responses. Fig. 1 shows the schema of the task consisting of 3 types of trials, randomly intermixed: no-signal trials (60\%), stop-signal trials $(20 \%)$, and ignore-signal trials (20\%).

Each trial started with the appearance of a central target (CT) (red circle, diameter $1.9 \mathrm{~cm}$ ) and a Cue signal (red circle, diameter $.7 \mathrm{~cm})$, smaller and appearing slightly above $(3 \mathrm{~cm})$ the CT. The monkeys had to touch the CT and keep their finger on it for various holding times (500-800 msec, 50-ms step). Subsequently, a peripheral target (PT) (red circle, diameter $1.9 \mathrm{~cm}$ ) appeared randomly at 1 of 2 possible locations (i.e., $7 \mathrm{~cm}$ to the right or left of the vertical midline of the screen; right only in 4/5 sessions Monkey 2). In all trials, after a foreperiod delay (variable duration, see Table 1) the Cue turned green (Go signal) (RGB: [0 250 0]; $85 \mathrm{~cd} / \mathrm{m}^{2}$ ) asking the subjects to respond (reaction time, RT; upper limit: $1200 \mathrm{msec}$ ). In nosignal trials, after the movement onset, the animal was required to maintain the new hand position for a variable time (600-800 msec, 50-ms step) until the end of the trial. In stopsignal trials, the Stop signal [blue circle, .7 cm RGB: (0 0 188), $70 \mathrm{~cd} / \mathrm{m}^{2}$ ] replaced the Go signal at the end of a variable and unpredictable interval (stop-signal delay, or SSD) during RT. In these trials, a hand that was kept on the CT until the end of the trial (800-1000 msec, 50-ms step) corresponded to a correct response (signal-inhibit trials). Conversely, the simple detachment of the hand after the presentation of the Go signal was defined as an incorrect response (signal-respond trials).
Similarly, in ignore-signal trials, the Ignore signal [yellow circle, $.7 \mathrm{~cm}$ RGB: (255 255 0), $65 \mathrm{~cd} / \mathrm{m}^{2}$ ] replaced the Go signal after a variable interval (ignore-signal delay or ISD) during RT. In these trials, the monkeys were instructed to respond as they did during no-signal trials, ignoring the Ignore signal. We defined ignore-RT (i-RT) as the time between the presentation of the Go signal and the onset of the hand movement, regardless of the appearance of the Ignore signal. If the onset of the hand movement occurred during the ISD (i.e., before the appearance of the Ignore signal), the trial was still considered to be a correct trial. Further, we defined ignore-signal RT (is$\mathrm{RT}$ ) as the time between the presentation of the Ignore signal and the onset of the hand movement. No upper RT was set in ignore-signal trials.

At the end of correct trials, the monkeys experienced a brief sound that was accompanied by the delivery of juice as a reward. In signal-respond trials (errors), neither sound nor reward was delivered, and the screen turned blank. The intertrial interval was set to $1000 \mathrm{msec}$.

Various fore-period delays (ranging from zero $\mathrm{ms}$, nodelay, to $1150 \mathrm{msec}$ ) were used in different sessions (for further details, see Table 1). Our purpose was to tentatively affect the level of movement preparation and the strategy that was used consequently. In humans, subjects adopt different strategies in the Selective stop task by manipulating other factors that influence the movement preparation, such as the relative proportion of stop versus ignore trials (Bissett \& Logan, 2014).

During stop-signal trials, a staircase procedure was adopted to determine the duration of the SSDs for each trial as follows: if the monkey succeeded in withholding the response, the SSD increased by 1 step ( $100 \mathrm{msec}$ ) in the subsequent stopsignal trial; conversely, if the subject failed, the SSD decreased by 1 step. The SSDs ranged from $120 \mathrm{msec}$ to $1020 \mathrm{msec}$, 
Table 1 - Details of behavioral performance from all sessions. (*) Sessions included in the neuronal analysis.

\begin{tabular}{|c|c|c|c|c|c|c|c|c|c|c|}
\hline $\begin{array}{l}\text { Monkeys } \\
\text { Sessions }\end{array}$ & $\begin{array}{c}\mathrm{n} \\
\text { trials }\end{array}$ & $\begin{array}{l}\text { Fore-period delay range } \\
\text { (50 msec step) }\end{array}$ & $\begin{array}{l}\text { no-signal } \\
\text { RT } \\
\text { Mean(SD) }\end{array}$ & $\begin{array}{c}\mathrm{n} \text { missed } \\
\text { no-signal } \\
\text { trials; } \mathrm{n}(\%)\end{array}$ & $\begin{array}{l}\text { signal- } \\
\text { respond RT } \\
\text { Mean(SD); } \\
\text { p }\end{array}$ & $\begin{array}{c}\text { Ignore-RT } \\
\text { Mean(SD); } \\
\text { p }\end{array}$ & $\mathrm{P}$ (Resp) & $\begin{array}{l}\text { median } \\
\text { SSD (s) }\end{array}$ & $\begin{array}{l}\text { SSRT } \\
(\mathrm{ms})\end{array}$ & Strategy \\
\hline M1.1* & 751 & $800-1150$ & 649 (144.4) & $1(.23)$ & $\begin{array}{l}542.3(128.8) \\
p<.001\end{array}$ & $\begin{array}{l}646.7(83.2) \\
p=.87\end{array}$ & .45 & .420 & 218 & DTS \\
\hline M1.2* & 1016 & $800-1150$ & 595.4 (187.5) & $7(1.2)$ & $\begin{array}{l}492(155) \\
p<.001\end{array}$ & $\begin{array}{l}571.8(216.5) \\
p=.13\end{array}$ & .39 & .320 & 224 & DTS \\
\hline M1.3 & 756 & $800-1150$ & 657.9 (145.8) & $3(.65)$ & $\begin{array}{l}555.8(125) \\
p<.001\end{array}$ & $\begin{array}{l}667.3(152.4) \\
p=.5\end{array}$ & .41 & .420 & 193 & DTS \\
\hline M1.4 & 894 & 0 & 844.7 (120.2) & $42(7.7)$ & $\begin{array}{l}808.7(120.6) \\
p=.01\end{array}$ & $\begin{array}{l}874.3(194.3) \\
p=.004\end{array}$ & .46 & .620 & 227 & STD \\
\hline M1.5* & 1210 & 0 & 819.5 (117) & $42(5.7)$ & $\begin{array}{l}785.8(135.2) \\
p=.005\end{array}$ & $\begin{array}{l}861.1(168.3) \\
p<.001\end{array}$ & .47 & .620 & 236 & STD \\
\hline M1.6* & 1311 & 0 & 908.6 (132.7) & $50(6.2)$ & $\begin{array}{l}853.8(136.9) \\
p<.001\end{array}$ & $\begin{array}{l}956.8(141.4) \\
p<.001\end{array}$ & .48 & .720 & 223 & STD \\
\hline M1.7 & 1457 & $320-570$ & 957 (160.3) & $21(2.4)$ & $\begin{array}{l}871.3(157) \\
p<.001\end{array}$ & $\begin{array}{l}985.2(142) \\
p=.01\end{array}$ & .47 & .721 & 218 & STD \\
\hline M1.8* & 1336 & $320-570$ & 968.6 (159.6) & $28(3.5)$ & $\begin{array}{l}935.4(149.1) \\
p=.02\end{array}$ & $\begin{array}{l}998(146.5) \\
p=.01\end{array}$ & .48 & .720 & 200 & STD \\
\hline M1.9 & 1375 & $320-570$ & $908(143.8)$ & $20(2.4)$ & $\begin{array}{l}841.8(108.5) \\
p<.001\end{array}$ & $\begin{array}{l}946.6(144.7) \\
p<.001\end{array}$ & .46 & .720 & 226 & STD \\
\hline M1.10 & 1311 & 0 & 786.1 (154) & $42(5.3)$ & $\begin{array}{l}727.4(132.2) \\
p<.001\end{array}$ & $\begin{array}{l}791.1(176.2) \\
p=.67\end{array}$ & .46 & .620 & 199 & DTS \\
\hline M2.1 & 752 & $800-1150$ & 659.4 (209.4) & $21(4.4)$ & $\begin{array}{l}558(144) \\
p<.001\end{array}$ & $\begin{array}{l}664 \text { (223.8); } \\
p=.6\end{array}$ & .48 & .420 & 236 & DTS \\
\hline M2.2* & 828 & $800-1150$ & 679.6 (192.4) & $23(4.4)$ & $\begin{array}{l}590.5(130.2) \\
p<.001\end{array}$ & $\begin{array}{l}703.7 \text { (214.5); } \\
p=.2\end{array}$ & .46 & .420 & 215 & DTS \\
\hline M2.3* & 1045 & $800-1150$ & 600.2 (149) & $9(1.4)$ & $\begin{array}{l}544.2(90.6) \\
p<.001\end{array}$ & $\begin{array}{l}637(174.7) \\
p=.003\end{array}$ & .49 & .420 & 204 & STD \\
\hline M2.4 & 497 & $800-1150$ & 583 (189.5) & $11(3.4)$ & $\begin{array}{l}508(117.2) \\
p=.016\end{array}$ & $\begin{array}{l}615(191.1) \\
p=.16\end{array}$ & .44 & .320 & 184 & DTS \\
\hline M2.5* & 944 & $800-1150$ & 405 (149) & $33(5.8)$ & $\begin{array}{l}337(121.3) \\
p<.001\end{array}$ & $\begin{array}{l}406(169) \\
p=.96\end{array}$ & .56 & .320 & 216 & DTS \\
\hline
\end{tabular}

depending on the performance. For each ignore-signal trial, the ISD was set to the SSD in the preceding stop-signal trial. The same procedure has been adopted in previous studies on human subjects (Bissett \& Logan, 2014).

\subsection{Behavioural analysis}

We analysed the monkeys' behavioural performance in the framework of the race model (Logan \& Cowan, 1984) to derive the SSRT. The race model assumes that during stop-signal trials, 2 stochastic processes race toward a threshold-the GO and STOP processes-triggered by the appearance of the Go and Stop signals, respectively. The movement generation or movement inhibition depends on which of these processes reaches its threshold first.

The main assumption of the race model is that the GO and STOP processes are independent of each other (independence assumption). As initially suggested (Logan \& Cowan, 1984), the race model can be used to estimate the SSRT in sessions where the independence assumption is validated, i.e., RTs in signal-respond trials must be shorter than RTs in no-signal trials, which means that the STOP process is unaffected by the presence of the Go signal.

We used the integration method to obtain the SSRT, since previously demonstrated as a solid method in sessions with a staircase approach to select SSDs (Band, van der Molen, \&
Logan, 2003); we first multiplied the number of no-signal trials by the average $\mathrm{P}($ Resp). The resulting value corresponded to the $n$th RT along the distribution of the no-signal RTs ordered by their duration. Then, the mean SSD is subtracted to the value of the $n$th RT, obtaining the SSRT. Following recent suggestions (Verbruggen et al., 2019), we also considered nosignal misses trials (no-signal trials with no response after the Go signal) when estimating the SSRT: we attributed to each no-signal misses trial the longer RT of no-signal trials, then we added these values to the no-signal trials distribution.

In the selective stop task, we assigned a behavioural strategy to each session as previously proposed (Bissett \& Logan, 2014; Sebastian et al., 2017) by comparing no-signal RTs and ignore-RTs. A stop-then-discriminate (STD) strategy was assigned if the ignore-RTs were slower than no-signal RTs. Conversely, discriminate-then-stop (DTS) strategy was assigned if the ignore-RTs were not slower than no-signal RTs. The rationale for these strategies is as follows: in the STD strategy, the subjects suppress their responses on the appearance of a signal that follows the Go signal and then restart the movement process after detecting it as an Ignore signal; in the DTS, the subjects first discriminate between signals (Ignore versus Stop), suppressing the response only on detection of a Stop signal. To provide statistical support for the assignment of categories, we performed t-test between the RTs of various types of trials and then applied Jeff Rouder's 
Bayes factor calculator (http://pcl.missouri.edu/bayesfactor)i.e., we converted t-value and sample size to a Bayes factor, thereby obtaining the odds in favour of the null or alternative hypothesis.

We also analysed the distribution of ignore-signal RTs by Hartigan's Dip Test of Unimodality.

\subsection{Neural recordings and analysis}

Unfiltered raw activity was recorded from 96 channels using a Utah array (Blackrock Microsystems, USA) and a TDT System 3 (Tucker Davies Technologies, sampling rate $24.4 \mathrm{kHz}$ ). From the raw signal that was recorded from each channel (site), we extracted the spectral estimate of the multiunit activity (MUA) offline as an approximation of the average firing rate, as described in (Mattia et al., 2013). The MUA was smoothed using a moving average sliding window ( \pm 20 -ms sliding window, 5-ms step).

From each recording session, we selected the sites with significant difference ( $t$-test, $p<.05$ ) between baseline (from 200 to $400 \mathrm{msec}$ following the touch of the CT) and the activity during at least 1 of 3 relevant trial events (the $200 \mathrm{msec}$ following the Go signal in no-signal and stop-signal trials; from 250 to $50 \mathrm{msec}$ before the movement onset in no-signal trials and the $200 \mathrm{msec}$ following the Stop signal onset in stop-signal trials). We considered the activity that was recorded from these sites to be task-related.

We then compared the activity between signal-inhibit trials and latency-matched no-signal trials. The latter comprised nosignal trials in which RTs were longer than the sum of the average SSD and the SSRT that was computed in the same session and had a similar level of movement preparation as in signal-inhibit trials. Latency-matched trials are trials in which, given the duration of the SSRT, the movement would have been cancelled by a Stop signal presented at the average SSD.

To determine whether a difference in MUA occurred between these 2 trial types, we applied the shuffle test for each interval, from $50 \mathrm{msec}$ before to $250 \mathrm{msec}$ after the onset of the Stop signal. In latency-matched no-signal trials, activity was aligned to the hypothetical presentation of the Stop signal (i.e., Go signal presentation + SSD); a method similar to others previously used (Mallet et al., 2016; Schmidt et al., 2013). First, for each interval, we compared the mean of the 2 trial types. Then, we shuffled the 2 trial types 10000 times for each interval, and for each shuffle, we compared the means of the 2 resulting trial distributions. We acquired a $\mathrm{P}$ value by counting the number of shuffles in which the difference between the obtained means was larger (or smaller) than that between the 2 observed means. We used a P value of .05 to define whether the difference between signal-inhibit trials and latencymatched no-signal trials was significant.

Thus, for each interval, we considered only sites for which were more than 9500/10 000 different means. Then, we performed the binomial test $(p=.05)$ to set a threshold and determine whether the measured fraction of sites was statistically significant for each interval.

At the end of this analysis, we selected the sites with activity that participated in inhibitory control-i.e., sites with activity that exceeded the baseline threshold for at least 50 msec during the SSRT.
The same method was used for comparisons of latencymatched no-signal trials and signal-inhibit trials with ignore-signal trials. When the ignore-signal trials were used, the activity was aligned to the presentation of the Ignore signal.

\subsection{Principal components analysis (PCA)}

To describe the multisite neuronal dynamics that were related to various behavioural strategies, we transformed the neuronal data by principal component analysis (PCA). We created a matrix $\mathrm{N}$ (recording sites) x $\mathrm{P}$ (MUA time value) by concatenating the average MUA across no-signal, ignoresignal, and signal-inhibit trials for each site. The MUA was aligned to the onset of the Stop signal (signal-inhibit trials), Ignore signal (ignore-signal trials), and to the hypothetical time of presentation of the Stop signal (latency-matched nosignal trials). The MUA spanned the interval from the average presentation of the Go signal to $800 \mathrm{msec}$ following the time of alignment.

We represented the evolution of neuronal dynamics as neuronal trajectories in 3D, in which the 3 dimensions were the first 3 principal components that explained most of the variance (at least 90\%). We reported the analysis separately for each recording session, with 1 exception: we combined the activity of 2 sessions from Monkey 2 when the DTS strategy was adopted, to increase the sample size and the reliability of the data. In these 2 sessions, to avoid oversampling from the same population of neurons, we selected only activity derived from different sites or, when from the same site, activity with different patterns in relation to movement generation and inhibition (see Supplementary Fig. 2).

To quantify the divergence between neuronal trajectories across trials that were related to movement inhibition, we measured the temporal evolution of the first derivative of the projection of the neuronal trajectory onto the single dimensions. For each dimension, we first defined the Neuronal Signal Time (NST) as the time in which the evolution of the projection changed direction after the Stop signal (signalinhibit trials) or the Ignore signal (ignore-signal trials)-i.e., the time in which the derivative was equal to 0 . Then, we calculated the weighted average of the resulting NSTs, based on the weight of the explained variance for each PCs.

\subsection{Relationship between neuronal activity and behavioural strategy}

The definition of the strategy adopted by each animal, for the criteria proposed by Bissett and Logan (2014), is possible only at the time when the animals generate the movement. To predict the strategy before that time, we looked at the differences in neuronal activity between the trial types using two classes of comparisons. Specifically we compared either latency-matched no-signal trials or signal-inhibit trials to ignore-signal trials, from $-50 \mathrm{msec}$ to $+225 \mathrm{msec}$ relative to the time of the relevant Stop/Ignore signal presentation.

For each recording site and class of comparison we derived (5 msec step) a squared Euclidean distance (Ed) from the neuronal activity in randomly generated pairs of trials (1000 total comparisons for each class; groups based on $75 \%$ of the 
total number of trials in the smallest category, to obtain the same numerosity). Then, we averaged among recording sites to obtain a distribution of 1000 values of average Ed for each time bin. Finally, we used a linear regression to obtain a slope in a sliding window interval of $50 \mathrm{msec}$ (step $5 \mathrm{msec}$ ). The slope indicates a measure of the trend (increasing/decreasing) for each comparison. By comparing slopes distributions at each temporal step (Kolmogorov-Smirnov test) we expect to be able to obtain a measure of the similarity/difference in the dynamics of the comparisons. This form of decoding should be able to detect when neuronal activities in the latencymatched no-signal trials versus ignore-signal trials comparison diverge from the signal-inhibit trials versus ignore-signal trials comparison, thus estimating when the STD strategy is being implemented.

The advantage of this method, in comparison to other classifiers, is that does not require a training period.

\section{Results}

\subsection{Behavioural results}

We derived behavioural data from 15 sessions (see Table 1; Monkey 1, 10 sessions; Monkey 2, 5 sessions) with a sufficient number of trials for analysis.

We found that monkeys adopted either the DTS or the STD strategy on different days. Consistent with the independence assumption of the race model, in both strategies, the SSRT divides the distribution of RTs in no-signal trials (Fig. 2, A shows 2 sample sessions; 1 for each strategy for the same animal) for fast (left portion) and slow (right portion) responses. As expected, signal-respond RTs overlapped primarily with fast responses in both strategies and are thus shorter than no-signal RTs (Fig, 2, B; Table 1).

Conversely, the distribution of responses in the ignoresignal trials showed strategy-specific differences. In the same sample session, the histograms in the lower area (Fig. 2, D) show that in the STD strategy, a bimodal distribution emerged in the is-RTs $[\mathrm{p}(\max )<.001]$. This pattern suggests that in certain trials (red bars), the Ignore signal activated behaviourally relevant inhibitory motor control, as confirmed by the longer duration of i-RTs compared with no-signal RTs (Fig. 2, C). Similar results were observed in all STD sessions (Monkey 1, 6 sessions: i-RTs: mean $941 \mathrm{msec}$, SD 170; no-signal RTs: mean $906.7 \mathrm{msec}$, SD 151.4, $p<.001$, rank-sum test; Monkey 2, 1 session; see Table 1). The slow mode of is-RTs always resulted in their occurring after the SSRT (Fig. 2, D), strengthening the hypothesis that when the monkeys adopt the STD strategy, they first inhibit and then restart the response after discrimination of the Ignore signal. When the monkeys adopted the DTS strategy during the ignore-signal trials, we did not find any behavioural signs of inhibitory control that was driven by the Ignore signal (Fig. 2 for a sample session; see also Table 1). None of the is-RT distributions showed bi-modality [p(min) > .7], and further, we did not observe a longer duration of i-RTs compared with no-signal trial RTs (Monkey 1, 4 sessions: iRTs mean 676 msec, SD 197.2; no-signal RTs mean $683.1 \mathrm{msec}$, SD 177.9, $p=.35$; Monkey 2, 4 sessions: i-RTs mean 580 msec, SD 235.2; no-signal RTs mean 575 msec, SD 218.1, $p=.58$, rank-sum test).

Following the observation that the Ignore signal influences behaviour only when the STD strategy is adopted, we examined whether the length of the inhibitory process in stopsignal trials is affected by the behavioural strategy (see Table 1). We did not observed any differences between SSRTs as a function of behavioural strategy [Monkey 1, STD strategy: mean SSRT $=221.67 \mathrm{msec}, \mathrm{CI}=(208.93,234)$, DTS strategy: mean SSRT $=208.5 \mathrm{msec}, \mathrm{CI}=(184.88,232.12)$, rank-sum test $p=.1$; Monkey 2, STD strategy: SSRT $=203 \mathrm{msec}$, DTS strategy: mean SSRT $=212.75 \mathrm{msec}, \mathrm{CI}=(178.58,246.92)]$; the same results were obtained after combining the data from both monkeys [STD strategy: mean SSRT $=219.14 \mathrm{msec}$, $\mathrm{CI}=(207.17,231.11)$; DTS strategy: mean SSRT $=210.63 \mathrm{msec}$, $\mathrm{CI}=(196.21,225.4)$, rank-sum test $p=.22]$.

The behavioural definition of strategies is based on the decision matrix per Logan and Bisset (2014); when computing other metrics (see Supplementary Materials Fig. 3), the distinction remains, although a smoother transition between the 2 strategies can be observed.

We were unable to demonstrate a clear link between foreperiod duration and strategy. For example, Monkey 2 used an STD strategy with long fore-period delays, and Monkey 1 used a DTS strategy with zero delay (see Table 1).

In conclusion, the behavioural evidence suggests that the Ignore signal temporarily inhibits behaviour only when the STD strategy was adopted. Concurrently, the overall duration of the inhibitory process that is driven by the Stop signal does not appear to be influenced by the strategy.

\subsection{Neuronal results}

We were able to record reliable neuronal signals in 8 sessions. In the other sessions $(n=7)$, technical problems prevented us from adequately collecting raw signals to analyse.

In all selected sessions we first identified, among the task related sites, those with a modulation involved in movement inhibition (see 2.5). We were able to select up to 76 modulated sites per session, an amount of data similar to that we used to collect with many behavioral sessions and a single-electrode recording approach (see Mirabella et al., 2011). Fig. 3 (top row) shows the temporal evolution of the fraction of task related sites that discriminated between trials when the movement was generated (latency-matched no-signal trials) versus inhibited (signal-inhibit trials) in 4 representative sessions - 1 for each monkey and strategy (see Supplementary Fig. 4, for other sessions). Across sessions and monkeys, approximately $78 \%$ of task related sites (mean \pm SE: $80 \pm 4 \%$ Monkey 1 and $73 \pm 10 \%$ Monkey 2; $p<.05$, shuffle test) showed a significant ( $p<.05$, binomial test) difference between nosignal and signal-inhibit trials during the SSRT (Fig. 3, black bars; see 2.5 for further details).

Then, for each session, we evaluated the fraction of task related sites that modulated differently when ignore-signal trials are compared with either latency-matched no-signal trials or signal-inhibit trials respectively (Fig. 3, middle and bottom rows). These plots (see also middle and bottom rows in the Supplementary Figure 4) show that the different sites in the recording arrays display modulation specifically related to 

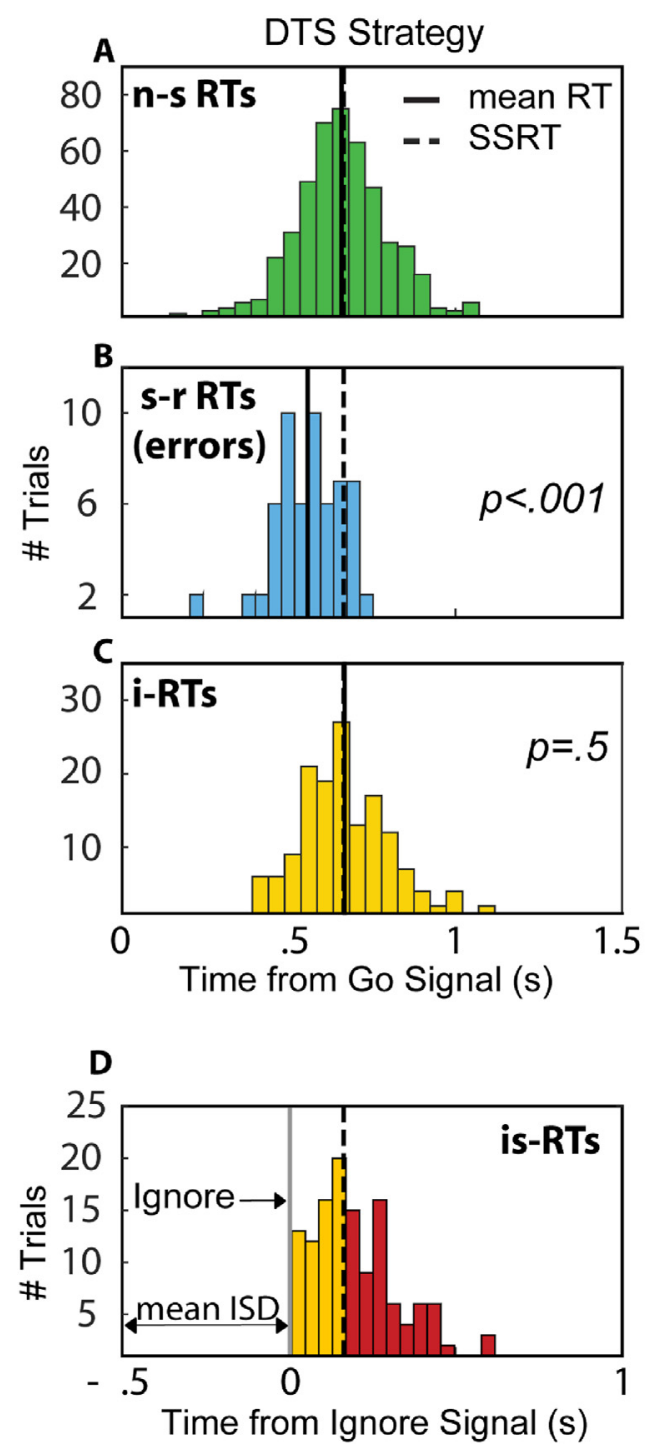
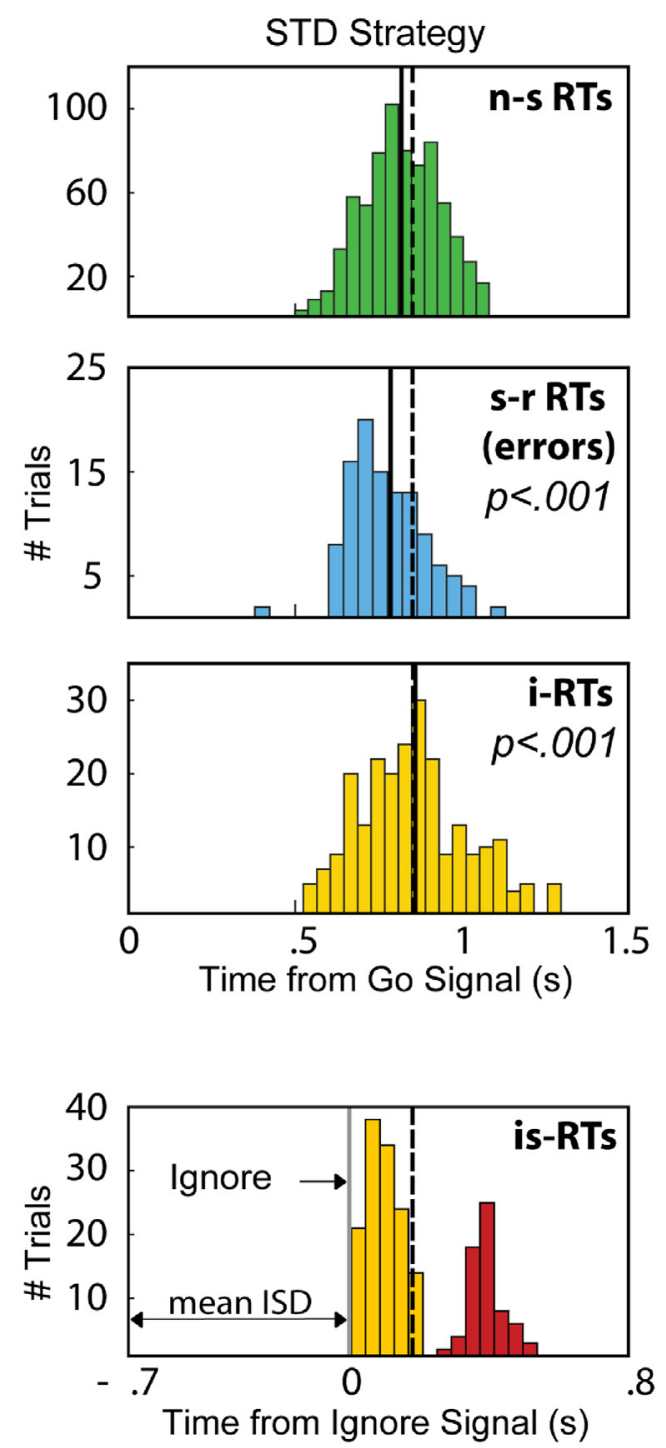

Fig. 2 - Reaction time distributions for both strategies. Each column shows data for the sessions classified by one of the two strategies (see 2.4). A, n-s, no-signal trial RTs. B, s-r, signal-respond trial RTs. C, ignore-signal trial RTs. In B and C, the P values are for the comparison (rank-sum test) with data in A. Continuous line: average RT; interrupted line: SSRT. D, same data in C aligned to the Ignore Signal (Ignore) and sorted by duration (in DTS, red bars are is-RTs > SSRT; in STD, red bars are those in the slow mode of the bi-modal distribution).

the strategy adopted by the animals. More in details, in DTS the pattern of modulated sites involved in motor inhibition (top row) was more similar to that observed for ignore-signal versus signal-inhibit trials comparisons (bottom row) than for ignore-signal versus latency-matched no-signal trials comparisons (middle row). In STD the pattern was the opposite.

Of relevance, these comparisons demonstrate that in the STD strategy, the MUA that followed the Ignore signals differed from the MUA that characterized movement execution (Fig. 3; middle-right plots)-similar to the activity that characterized movement inhibition-in most of the involved recording sites.

Moreover, in STD, Fig. 3 shows that the time at which a significant fraction of sites began to show a difference for ignore-signal versus latency-matched no-signal trials comparisons (bottom right panels) was delayed by up to $90 \mathrm{msec}$ (mean across sessions \pm SE: $48 \pm 18.3 \mathrm{msec}$ ) when compared to the time of significant modulation related to the inhibitory control (top right panels; see also data in Supplementary Fig. 4). This suggests that modulation is initially similar and then diverges.

Once we selected sites that participated in inhibitory control-i.e., sites that exceeded the threshold during the SSRT in the previous analysis-we tested whether an inhibitory process developed in the same sites following the Ignore signal by comparing the neuronal activity that was recorded in ignoresignal trials versus no-signal and signal-inhibit trials. The neuronal activity of no-signal trials characterizes the movement generation, whereas that in signal-inhibit trials defines movement suppression. In ignore-signal trials, a movement is generated, and the neuronal activity should be similar to that 
DTS
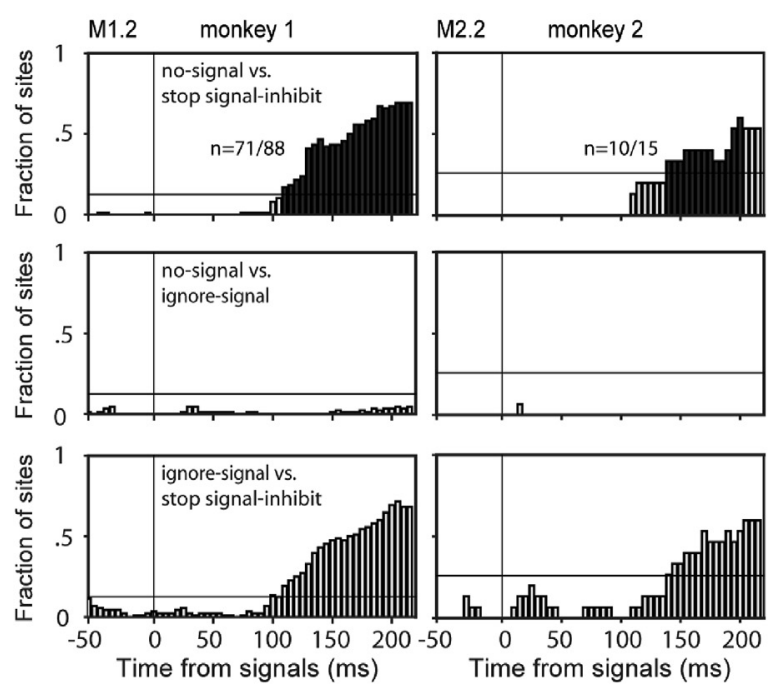
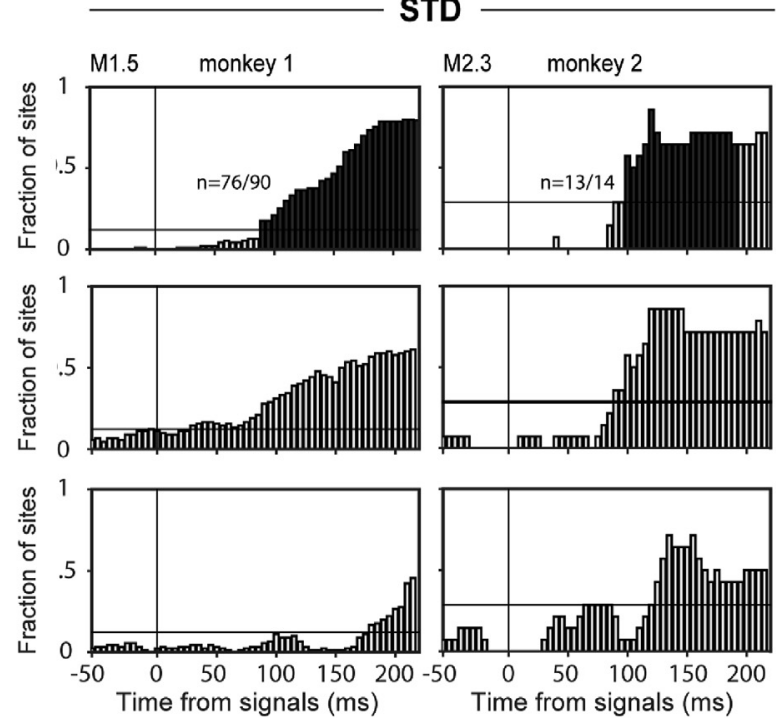

Fig. 3 - Neuronal characterization of behavioral strategies at the population level. Fraction of sites with significantly different MUAs for the trial types under comparison. Data are presented separately for each monkey and for the two strategies (DTS and STD). Each row shows the results of the comparisons indicated in the first panel. The alignment time is the Stop signal for signal-inhibit trials, the Ignore signal for ignore-signal trials, and the hypothetical (see 2.5) presentation of the Stop signal for latency-matched no-signal trials. The analysis was performed across all sites showing task-related activity (numbers are in the panels at the top of each column). The horizontal line on each plot corresponds to the threshold obtained from the shuffle test $(p<.05)$ between each pair of trials and the binomial test $(p=.05)($ see 2.5$)$. Dark bars in the top plots indicate the fraction of sites that significantly exceeded the threshold during the SSRT (see 2.5).

in no-signal trials. However, if an inhibitory process has been triggered, the ignore-signal activity should bear at least some resemblance to the activity in signal-inhibit trials. The behavioural data and the neuronal population analysis above suggest that this similarity should be observed only when the STD is adopted, at least for a short period of time after the presentation of the Ignore signal.

Figure 4 shows that the neuronal patterns expressed after the presentation of either the Stop signal or the Ignore signal are different in the two strategies. In the DTS strategy the only deviation observed is for the activity in the stop signal-inhibit trials. In the STD strategy a more complex pattern emerges: the activity in ignore-signal trials undergoes modulation that is similar to that in signal-inhibit trials before the end of the SSRT; subsequently the activity deviated again and displays a pattern similar to that in no-signal trials.

The initial strong similarity between the activity in ignoresignal trials and signal-inhibit trials in STD suggests temporary activation of the inhibitory process once a novel signal appears (either Stop or Ignore) after the Go signal.

To provide a representative neuronal dynamics at the population level we adopted a dimensionality reduction approach by principal component analysis (PCA; see 2.6). Across strategies, the neuronal activities that were extracted from the 3 trial types had similar evolution until the Stop/ Ignore signal presentation, for both monkeys (Fig. 5). After these events, the differences between strategies emerged. In both the strategies, the neuronal activity in signal-inhibit trials deviated from the trajectories of no-signal and ignoresignal trials, before the end of the SSRT (the duration is indicated as the thick portion in the trajectories), by traveling toward a subspace in which occupancy was unrelated to movement generation.

When monkeys adopted the DTS strategy (Fig. $5 \mathrm{~A}$ ) there is no evident effect of the appearance of the Ignore-Signal in ignore-signal trials. In contrast, when monkeys adopted the STD strategy (Fig. 5 B), the neuronal dynamics that followed the presentation of the Ignore signal initially underwent a similar evolution as that in signal-inhibit trials, moving toward another region of the space that was unrelated to the movement generation. After this first deviation, the trajectories shifted again, evolving as in no-signal trials (for other sessions, see Supplementary Figure 5).

Once we observed the neuronal dynamics that characterized the various behavioural strategies, we estimated the time that the trajectories deviated after the Stop and Ignore signals presentation. To this end, we evaluated the NST (Fig. 6), defined as the time at which the first derivative of the projection of the neural trajectory onto the single components crossed the zero line (see 2.6). The NST indicates when the trajectories change direction after the Stop or the Ignore signals. We found that the NST that characterized the inhibition in signal-inhibit trials occurred (mean across sessions \pm SE): $70 \pm 5 \mathrm{msec}$ (DTS strategy) and $82.5 \pm 10 \mathrm{msec}$ (STD strategy) after the Stop signal. In the DTS strategy (Fig. 6, top) the first derivative of the neuronal activity in all PCs shows the same pattern for both no-signal and ignore-signal trials. In contrast, when the STD was used (Fig. 6, bottom), the derivative in ignore-signal trials crossed the zero value twice after the Ignore signal, the first time in close proximity with the crossing observed in signal-inhibit trials. The first NST (mean across sessions \pm SE: $96.7 \pm 14.8 \mathrm{msec}$ ) reflects the time at 

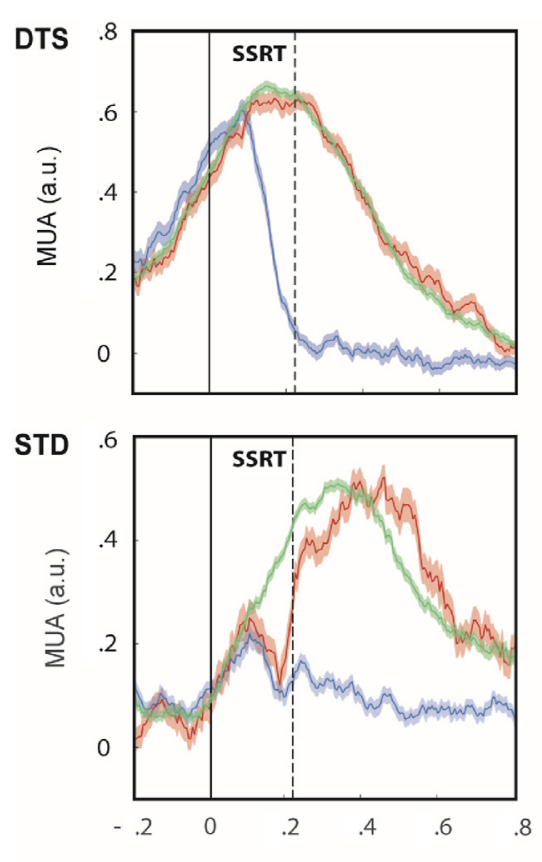

Time from Stop/lgnore signals (s)
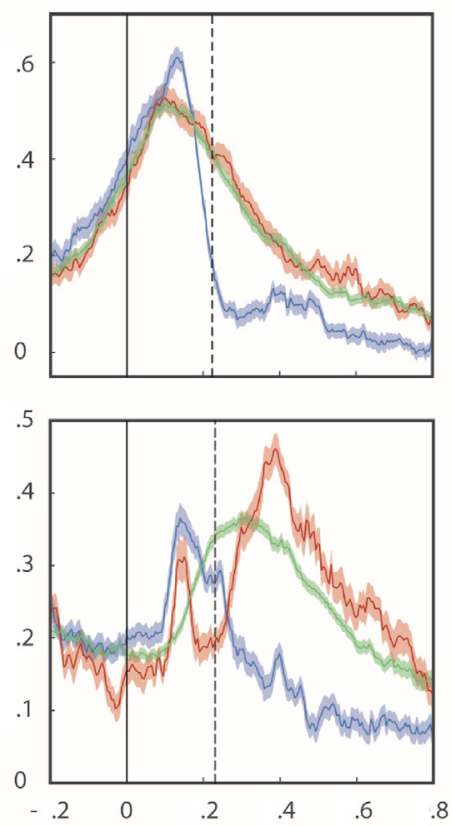

Time from Stop/lgnore signals $(s)$
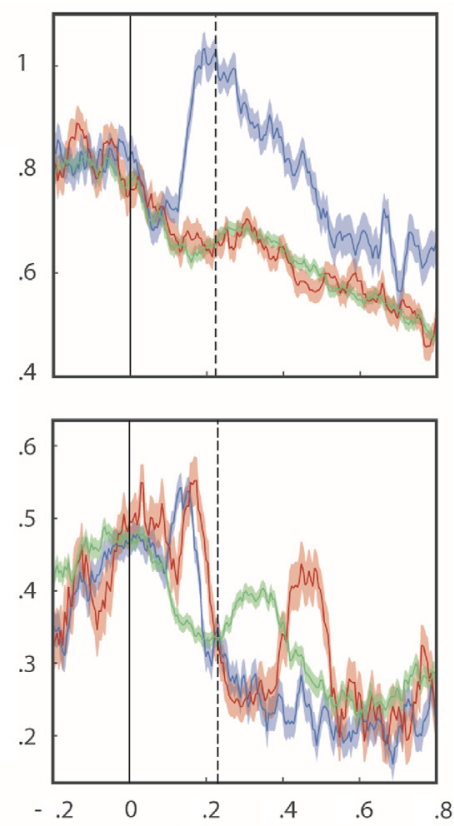

Time from Stop/lgnore signals (s)

- no-signal trials _ stop signal-inhibit trials _ ignore-signal trials

Fig. 4 - Examples of neuronal patterns for the 2 behavioural strategies. Average MUA ( \pm SE) from three sample sites are shown for each trial type and strategy (DTS and STD). Sites are examples of those extracted by the shuffling analysis depicted in Fig. 3. SSRT: stop signal reaction time.

which the trajectories in ignore-signal trials deviated from those in no-signal trials, starting the inversion that characterizes the inhibitory process, whereas the second NST (mean across sessions \pm SE: $158 \pm 7.8 \mathrm{msec}$ ) indicates the end of this temporary inhibition. Based on the difference in time between the 2 deviations, the duration of this temporary inhibitory process was approximately $60 \mathrm{msec}$ (mean across sessions \pm SE: $60.8 \pm 8.8 \mathrm{msec}$ ).

We also tested the relationship between the NST in signalinhibit trials and the SSRT as function of the behavioral strategy. For each session we calculated the time at which the NST occurred with respect to the end of the SSRT (i.e., SSRTNST). As a result, we found that this measure did not change based on the behavioral strategy [mean across sessions $\pm \mathrm{SE}$ : $138.7 \pm 10.9 \mathrm{msec}$ (DTS Strategy) and $141 \pm 4 \mathrm{msec}$ (STD Strategy), $p=1$, rank-sum test]. Given the stable relationship between the neuronal (NST) and behavioral (SSRT) processes we could consider the use of the NST, as an inhibitory index, in those cases in which the independence assumption is not satisfied and it is not possible to estimate the SSRT. This would be extremely interesting when other behavioural strategies are implemented (see 4).

Finally, we asked whether it is possible to extract a neuronal signature that allows one to classify the strategy before the motor response, i.e., the event that is necessary for strategy definition behaviourally (Bissett \& Logan, 2014).

To this end we focused on the $200 \mathrm{msec}$ following the Ignore signal and measured how the MUA differs when the movement is generated under various conditions (no-signal and ignore-signal trials) with respect to the activity when it is inhibited (signal-inhibit trials). For all of the sessions of the animal with a sufficient number of different sessions for the different strategies (Monkey 1) we performed 2 comparisons: latency-matched no-signal trials versus signal-inhibit trials and ignore-signal trials versus signal-inhibit trials. The trials were selected by employing the same criteria across sessions and the selection was blind respect to the behavioural strategy.

Figure 7 summarizes the analysis for 2 representative sessions-1 for each strategy (for other sessions see Supplementary Fig. 6). For each comparison (latency-matched no-signal trials versus signal-inhibit trials and ignore-signal trials versus signal inhibit trials), the distributions of slopes (top panels) for 2 sample time intervals, t1 (centered at the signal onset) and t2 (centered $150 \mathrm{msec}$ following the signal onset) and the averaged slopes over time (bottom panels) are shown. During the DTS strategy, movement generation was anticipated by a similar evolution of slopes when comparing the MUA in latency-matched no-signal trials and ignoresignal trials with that in signal-inhibit trials, demonstrating that in this case, movement preparation in ignore-signal trials is not influenced by the presence of the Ignore signal.

Conversely, during the STD strategy, movement execution was anticipated by a different evolution of slopes over time. The slopes separated between comparisons at approximately $100 \mathrm{msec}$ after presentation of the signal. After this time, the differences between latency-matched no-signal trials and signal-inhibit trials increase, due to the higher level of motor preparation in latency-matched no-signal trials compared with ignore-signal trials. This greater preparatory activity in 


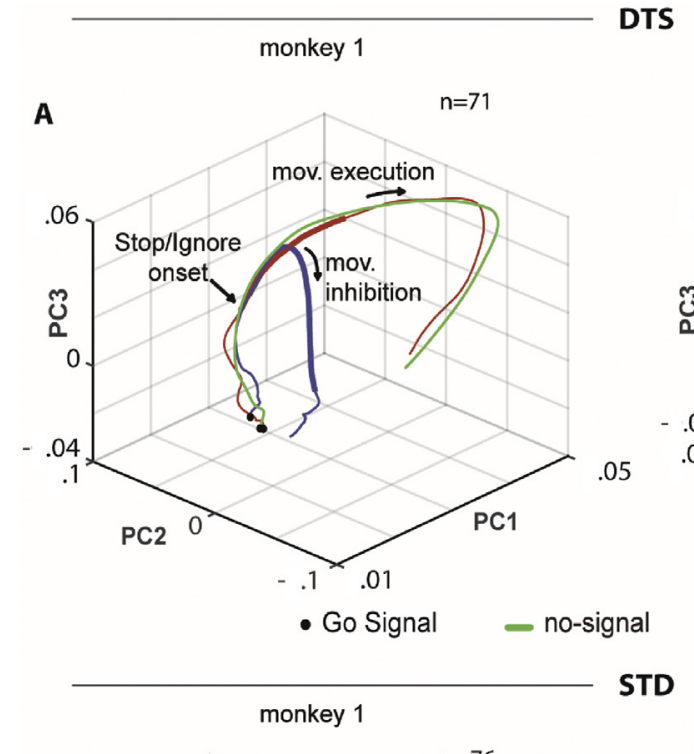

\section{DTS}
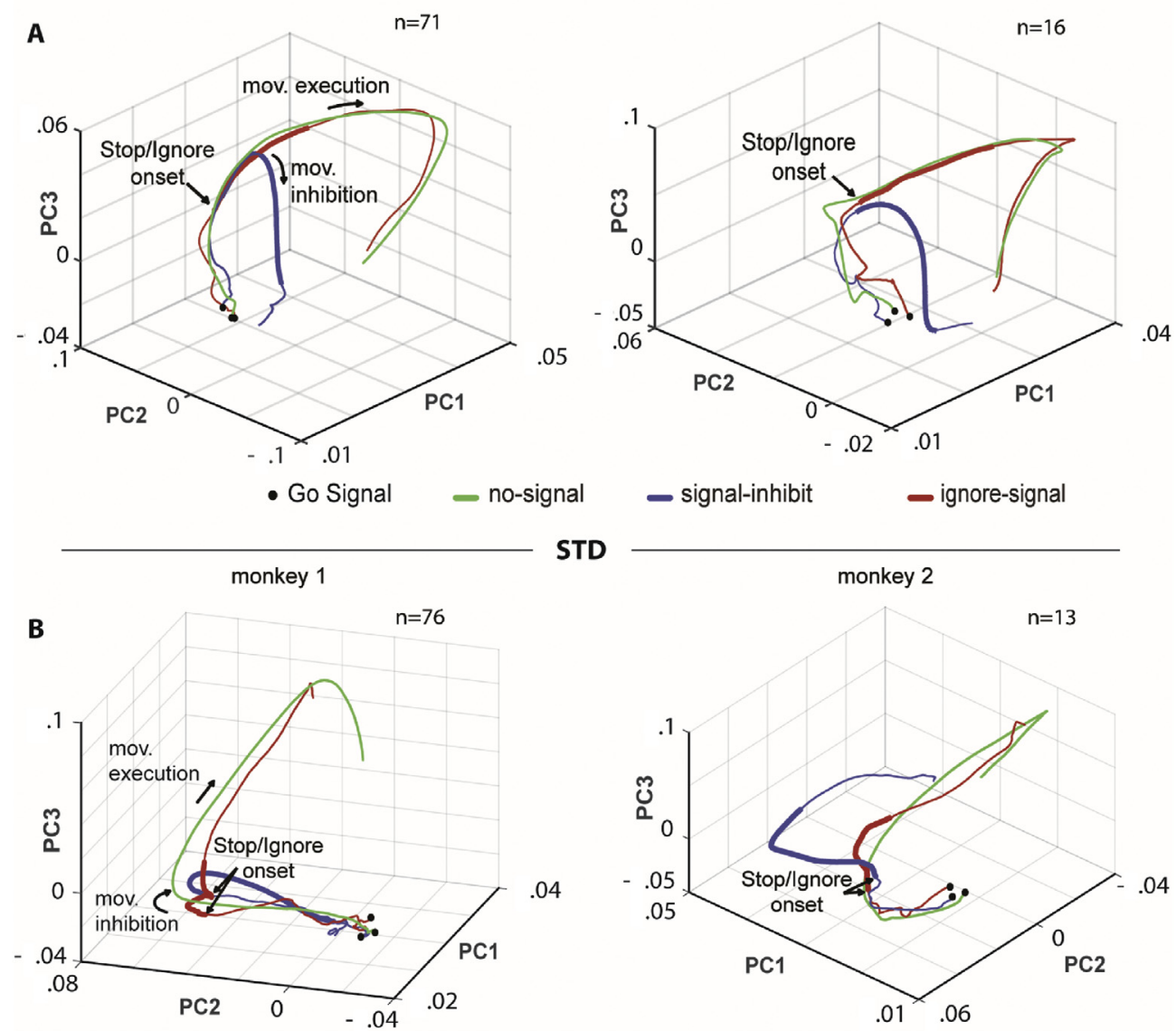

STD
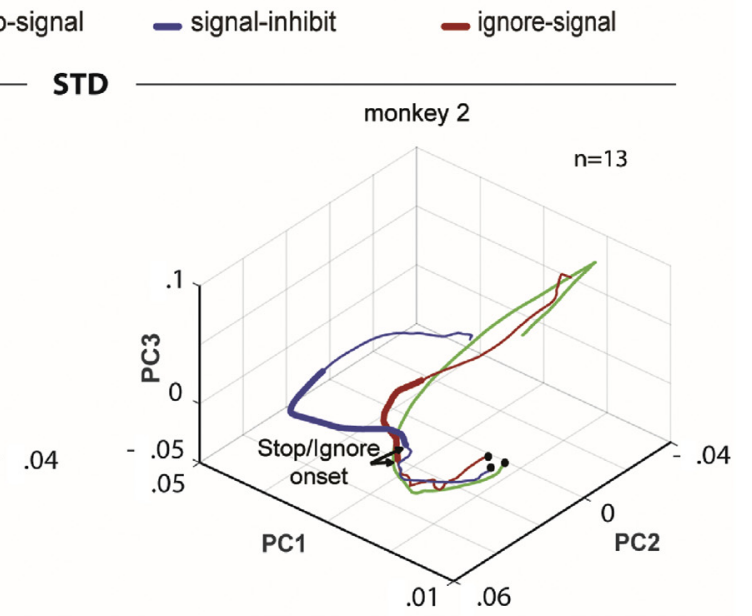

Fig. 5 - Population neuronal dynamics across animals and behavioural strategies. For each strategy, one session for each monkey is presented. Neuronal trajectories are aligned to the Stop signal for signal-inhibit trials, the Ignore signal for ignore-signal trials, and the hypothetical presentation (see 2.6) of the Stop signal in latency-matched no-signal trials, starting from the average presentation of the Go signal (black dots) to $800 \mathrm{msec}$ following the alignment. The thick portions of the trajectories represent the duration of the SSRT.

latency matched-no signal trials reflects the shorter RTs that will be observed in the DTS.

A Kolmogorov-Smirnov test was repeated for each time bin. Two separate sets of $p$ values emerged from the analysis. One set was composed of $p$ values $<.05$ (n $=3$ sessions, in all cases $p<.0001)$, and the other set comprised $p$ values $>.05(\mathrm{n}=2$ sessions, in all cases $p>.3)$. This classification was confirmed by hierarchical cluster analysis (see Supplementary Fig. 6, bottom). Then, we traced back to the behaviour of the sessions and found that sessions with $p$ value $<.05$ were associated with the STD strategy, whereas those with $p>.05$ correlated with the DTS strategy.

\section{Discussion}

We examined the neuronal instantiation of inhibition in the PMd using a stimulus selective stop task. This task allowed us to determine the influence of the behavioural strategy on the processing of the Ignore signal.
We found that monkeys performed the selective stop task using 2 strategies-STD and DTS-while solving the ignoresignal trials and that there was a strong correlation between the adopted strategy and the effect of the Ignore signal. Specifically, an inhibitory effect on neuronal activity was only observed when the subject implemented the STD strategy. As a result of applying a state-space approach, based on dimensionality reduction, this relationship was congruently established for the single-site- and multisite-level analyses. Consistent with these findings, we assert that the Ignore signal drove the movement inhibition, as represented in the neuronal dynamics of the PMd in a specific behavioural context.

In the state-space representation, movement is generated if the neuronal activity travels from a subspace that corresponds to the absence of arm movements to another subspace that will allow their initiation. Along this trajectory, the presentation of a Stop signal can affect the evolution of neuronal activity. In signal-inhibit trials, the neuronal evolution was halted and reversed following the Stop signal: the neuronal activity shifted toward the initial subspace or possibly toward 

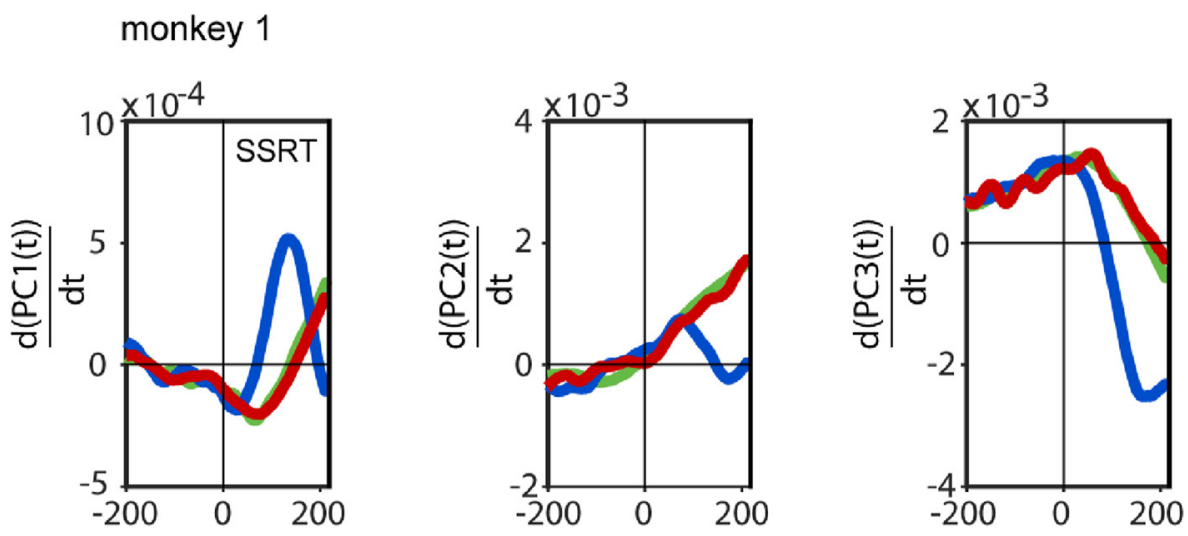

Time from Stop/lgnore (ms)

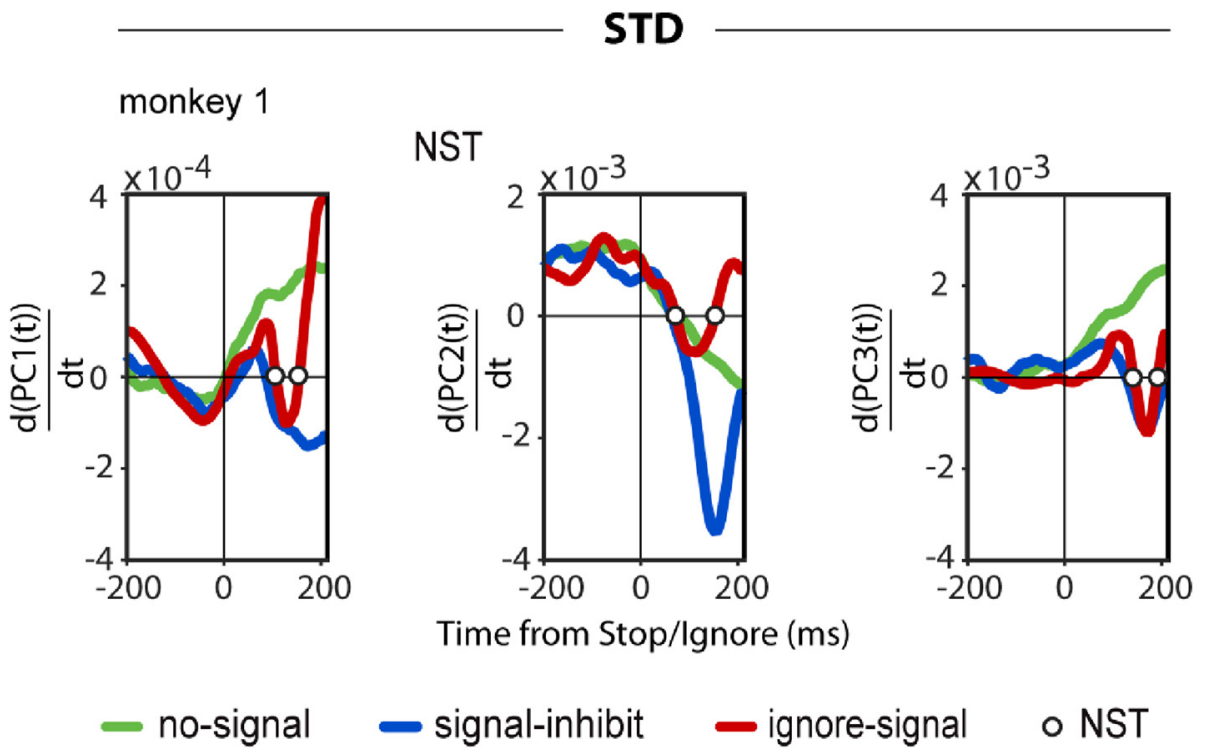

Fig. 6 - First derivative of principal components (PCs). Computation of the duration of the temporary inhibitory process. Data are from the same sessions in Fig. 5 for Monkey 1. White dots in the lower panels (STD) indicate the time of crossing of the zero line in ignore-signal trials. The distance between the two dots was used to estimate the duration of the deviation of the trajectory as consequence of the Ignore-signal presentation (see 2.6) in STD.

another subspace, the occupancy of which does not allow the initiation of the movement.

The emerging behavioural strategies are clearly represented in the neuronal dynamics. When the STD is implemented, in ignore-signal trials, the trajectories are initially affected similarly as in signal-inhibit trials: the neuronal dynamics momentarily reverse their trend, moving toward the initial subspace. However, subsequently, when the meaning of the Ignore signal is clarified, the trajectory follows the evolution that is observed in the no-signal trial, reaching the subspace that leads to initiation of the movement. When DTS strategy is used, the evolution in ignore trials is similar to that for no-signal trials: in this case, there is no evidence of the activation of the inhibitory process.

The observation that the initiation of arm movement requires a shift from one subspace to another is consistent with findings that were obtained with the reaching delay task and a dynamic system approach (Ames, Ryu, \& Shenoy, 2014; Kaufman et al., 2014; Shenoy, Sahani, \& Churchland, 2013). Several of these studies showed that movements are prepared during the delay epoch, in a subspace (output-null dimension) that prevents neuronal activity from affecting the muscle and generating movements. Movements are executed when neuronal trajectories reach another subspace (output-potent dimension), in which rotational dynamics appear (Churchland et al., 2012; Elsayed \& Cunningham, 2017). In our study, the neuronal activity remained in a subspace during the first period of all the trials and during the last period of the signal-inhibit trials (see also Pani et al., 2019), when movements are not generated.

As shown by the derivative analysis, when the STD is adopted in ignore-signal trials, the neuronal activity travels 

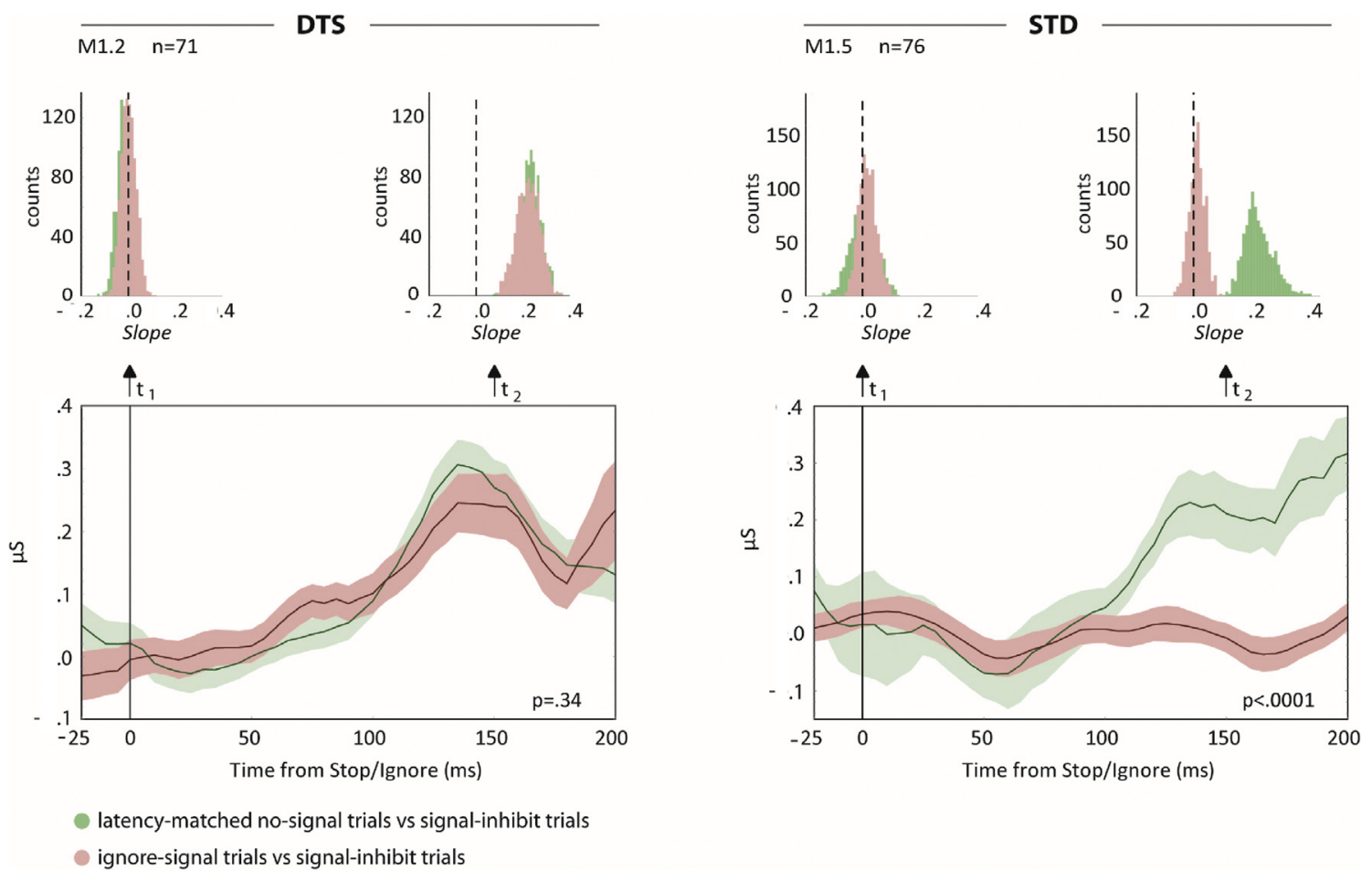

latency-matched no-signal trials vs signal-inhibit trials

ignore-signal trials vs signal-inhibit trials

Fig. 7 - Behavioural strategies are predicted by the neuronal activity shortly after the Ignore signal. Top: For each strategy, distributions of slopes of the Euclidian distances of neuronal activities computed for comparisons of no-signal trials versus signal-inhibit trials (red) and of ignore-signal trials versus signal-inhibit trials (green) at the signal onset (t1) and $150 \mathrm{msec}$ after signal onset (t2). Bottom: Evolution over time of the average slope $(\mu \mathrm{S})$ of the Euclidean distances for the same comparisons. The level of separation of the two curves is a good indicator of the difference between distributions (see 2.7). Data are from the same session in Fig. 5, left panels.

first toward the movement initiation subspace, reverses slightly, and then finally re-reverses to allow the movement generation.

This finding is the first demonstration of the dependence of movement inhibition on the adopted strategy. Following presentation of the Ignore signal, clear dynamics of inhibition emerged only in the STD strategy, correlating directly with behaviour and occurring before the behavioural strategy could be established.

To date, only one primate neurophysiology study (Xu et al., 2017) has investigated the neural mechanisms of selective inhibition in the saccadic system. In that study, the authors demonstrated a dissociable role of the right ventrolateral prefrontal cortex (rVLPFC) and FEF in response control. While the rVLPFC encodes information about the meaning of the signals (Stop signal versus Ignore signal), the FEF activity is related more to the decision to move or cancel the movement (successful versus unsuccessful inhibition) regardless the meaning of the signal.

Although we examined the neuronal dynamics that underlie movement inhibition using a state-space approach, the study of the neural basis of movement inhibition for other systems and structures has been oriented primarily toward the characterization of specific cell types. The combination of countermanding task and neurophysiology has yielded significant results for the saccadic system in monkeys and for the function of basal ganglia in movement inhibition in rats.
In the monkey saccadic system, movement control has been ascribed to 2 cell types in the frontal eye fields (FEF) and superior colliculus (SC): movement cells and fixation cells. Saccades are made when movement cells increase their firing activity, whereas fixation cells decrease it; saccades are inhibited when the opposite pattern occurs, following the Stop signal and before the end of the SSRT (Hanes et al., 1998; Paré \& Hanes, 2003). A recent study found that a subpopulation of dopaminergic neurons in the substantia nigra (SNc) and a connected subpopulation of striatal neurons fire differently when saccades are made versus withheld and before the end of the SSRT (Ogasawara, Nejime, Takada, \& Matsumoto, 2018). However, the relationship between nigro-striatal, cortical, and collicular activities must be clarified.

Clear neuronal types that participate in various aspects of movement inhibition have also been found in the basal ganglia of rats: a series of studies by Berke and colleagues (Schmidt et al., 2013; Mallet et al., 2016) have found that movement inhibition can be mapped to different cell types and structures. When a Stop signal occurs, movements are first paused briefly by the neuronal activity in the subthalamic nucleus and substantia nigra and then cancelled, if necessary, by neurons in the pallidus, affecting the striatum (Schmidt \& Berke, 2017). This modulation purportedly affects the neuronal dynamics in cortical motor regions.

It is not possible to establish the definitive relationship between neuronal types and aspects of movement inhibition for the limb cortical motor system in primates (Kaufman et al., 
2010). Single cells in the PMd show are heterogeneous, preventing any simple or mechanistic classification, as can be performed for the saccadic system and basal ganglia (Kaufman et al., 2010; Mirabella et al., 2011). In our study, we could not execute this classification, because we recorded the spectral derived MUA, which the reflects spiking activity of small population of neurons that surround the tips of the electrodes-by their very nature, many neuronal types can contribute to the activity.

However, the patterns that we observed strongly resemble the typical patterns of activity in single cells, and most importantly, their function in movement inhibition is supported by their SNTs before the SSRT, as observed in other studies (Pani et al., 2018). The system-level implementation of movement inhibition requires communication between various regions, each of which can experience specific neuronal implementation of the inhibitory process, by specific neural type or population code (Aron et al, 2016; Pouget et al., 2011). Assuming that primates use a basal ganglia-based mechanism for control of limb movements, similar to that in rodents, the modulations during movement inhibition that are recorded in the basal ganglia (and in other regions) might appear to be heterogeneous when viewed at the motor cortical level (Mattia et al., 2013; Oldenburg \& Sabatini, 2015), thus rendering the state-space approach a suitable method for describing the inhibition of limb movements.

This study strengthens the evidence in favour of the PMd as a site of movement control. It is well established that the PMd continuously signals the momentary decision state about forthcoming movements (Kaufman et al., 2014; Thura \& Cisek, 2014) and movement parameters (velocity, reaction time). The data that support its function in movement inhibition, as required by the stop-signal task, are accumulating (Bardella, Pani, Brunamonti, Giarrocco, \& Ferraina, 2020; Giamundo et al., 2019; Mattia, Ferraina, \& Del Giudice, 2010; Mirabella et al., 2011; Pani et al., 2013, 2018). In this study, we demonstrated that the function of the PMd in inhibition is strategydependent-i.e., the PMd reflects movement inhibition only when it is behaviourally relevant. The presentation of the Ignore signal does not drive the inhibition per se but only under the conditions in which the signal can influence the movement plan. Further, by using the stop task, movement inhibition-related activity is clearly represented in PMd neurons (Mirabella et al., 2011; Pani et al., 2018), whereas attempts to detect coherent activation in other premotor areas controlling limb movements and anatomically connected to PMd (Johnson \& Ferraina, 1996) have been unfruitful (Scangos \& Stuphorn, 2010).

In the literature on selective inhibition in humans (Bissett \& Logan, 2014), the DTS strategy is usually described as Independent DTS to distinguish it from a Dependent DTS strategy. The Dependent DTS strategy is characterized by signal-respond RTs that are no slower than no-signal RTs, thus violating the race-model independence assumption. In this last case, some form of inhibition is believed to occur through the trial, delaying the response in respond stop trials (Bissett \& Logan, 2014; Sebastian et al., 2017).
We did not find any behavioural evidence that could be referred to as Dependent DTS. One possible reason is that the monkeys were highly trained in performing the task and that the amount of training led them to develop a more efficient strategy in deciding between stopping and moving.

Moreover, Monkey 2 exhibited the STD strategy on only one day and it might be due to the number of sessions. We should consider the possibility that different subjects could have a preference for either the STD or the DTS strategy. This does not seem to be the case for Monkey 1, which alternated more regularly the two strategies. In any case, to test all possibilities we should have collected more sessions. However, the STD and DTS are also observed in humans (Bissett \& Logan, 2014; Sebastian et al., 2017). One additional caveat is that in the STD, the SSRT should be shorter than when the DTS is used (Bissett \& Logan, 2014). This difference is related to the presence of a longer discrimination stage following the Ignore signal in the Independent DTS. We did not observe such an effect after analysing our data: in general, the length of the SSRT did not differ between strategies. Further, analogous results have been observed in humans by similar studies (Bissett \& Logan, 2014; Sebastian et al., 2017). It should be noted that in our study we tested multiple times the same monkeys, while in human studies conducted so far, subjects have been tested only once. In future studies, it would be interesting to investigate whether humans can change strategy across days, like monkeys, as a result of a different implementation of the inhibitory process.

\section{Authors contribution}

F.G., P.P, and S.F. designed the experiment. F.G., F.F. prepared the experimental setup and collected the data. F.G. and G.B. analyzed the data. All authors participated in the interpretation of data and helped draft the manuscript; P.P. and S.F. supervised the project. All authors gave final approval for publication.

\section{Open practices}

The study in this article earned Open Materials and Open Data badges for transparent practices. Materials and data for the study are available at: https://osf.io/qfs3k/.

We report how we determined our sample size, all data exclusions, all inclusion/exclusion criteria, whether inclusion/exclusion criteria were established prior to data analysis, all manipulations, and all measures in the study.

No part of the study procedures neither study analyses were pre-registered prior to the research being conducted.

\section{Acknowledgments}

We thank Jeff Schall and one anonymous reviewer for constructive comments to the first draft of the manuscript. 
Partially supported by Sapienza (grant number PH117IC823A9528, 2017).

\section{Supplementary data}

Supplementary data to this article can be found online at https://doi.org/10.1016/j.cortex.2020.09.032.

\section{R E F E R E N C E S}

Ames, K. C., Ryu, S. I., \& Shenoy, K. V. (2014). Neural dynamics of reaching following incorrect or absent motor preparation. Neuron, 81, 438-451. https://doi.org/10.1016/ j.neuron.2013.11.003

Aron, A. R. (2010). From reactive to proactive and selective control: Developing a richer model for stopping inappropriate responses. Biological Psychiatry, 69(12), e55-68. https:/doi.org/ 10.1016/j.biopsych.2010.07.024

Aron, A. R., Durston, S., Eagle, D. M., Logan, G. D., Stinear, C. M., \& Stuphorn, V. (2007). Converging evidence for a fronto-basalganglia network for inhibitory control of action and cognition. The Journal of Neuroscience, 27(44), 11860-11864. https://doi.org/ 10.1523/JNEUROSCI.3644-07.2007

Aron, A. R., Fletcher, P. C., Bullmore, E. T., Sahakian, B. J., \& Robbins, T. W. (2003). Stop-signal inhibition disrupted by damage to right inferior frontal gyrus in humans. Nature Neuroscience, 6(2), 115-116. https://doi.org/10.1038/nn1003

Aron, A. R., Herz, D. M., Brown, P., Forstmann, B. U., \& Zaghloul, K. (2016). Frontosubthalamic circuits for control of action and cognition. The Journal of Neuroscience, 36(45), 11489-11495. https://doi.org/10.1523/JNEUROSCI.2348-16.2016

Aron, A. R., \& Poldrack, R. A. (2006). Cortical and subcortical contributions to stop signal response inhibition: Role of the subthalamic nucleus. The Journal of Neuroscience, 26(9), 2424-2433. https://doi.org/10.1523/JNEUROSCI.4682-05.2006

Band, G. P., van der Molen, M. W., \& Logan, G. D. (2003). Horserace model simulations of the stop-signal procedure. Acta Psychologica, 112(2), 105-142. https://doi.org/10.1016/s00016918(02)00079-3

Bardella, G., Pani, P., Brunamonti, E., Giarrocco, F., \& Ferraina, S. (2020). The small scale functional topology of movement control: Hierarchical organization of local activity anticipates movement generation in the premotor cortex of primates. Neuroimage, 207, 116354. https://doi.org/10.1016/ j.neuroimage.2019.116354

Bissett, P. G., \& Logan, G. D. (2014). Selective stopping? Maybe not. Journal of Experimental Psychology General, 143(1), 455-472. https://doi.org/10.1037/a0032122

Boehler, C. N., Appelbaum, L. G., Krebs, R. M., Chen, L. C., \& Woldorff, M. G. (2011). The role of stimulus salience and attentional capture across the neural hierarchy in a stopsignal task. Plos One, 6(10), 1-10. https://doi.org/10.1371/ journal.pone.0026386

Brunamonti, E., Ferraina, S., \& Paré, M. (2012). Controlled movement processing: Evidence for a common inhibitory control of finger, wrist, and arm movements. Neuroscience, 215, 69-78. https://doi.org/10.1016/j.neuroscience.2012.04.051

Brunamonti, E., Pani, P., Papazachariadis, O., Onorati, P., Albertini, G., \& Ferraina, S. (2011). Cognitive control of movement in Down syndrome. Research in Developmental Disabilities, 32(5), 1792-1797. https://doi.org/10.1016/ j.ridd.2011.03.008

Cai, W., Oldenkamp, C. L., \& Aron, A. R. (2011). A proactive mechanism for selective suppression of response tendencies.
The Journal of Neuroscience, 31(16), 5965-5969. https://doi.org/ 10.1523/JNEUROSCI.6292-10.2011

Chikazoe, J., Jimura, K., Asari, T., Yamashita, K., Morimoto, H., Hirose, S., et al. (2009). Functional dissociation in right inferior frontal cortex during performance of go/no-go task. Cerebral Cortex, 19(1), 146-152. https://doi.org/10.1093/cercor/bhn065

Churchland, M. M., Cunningham, J. P., Kaufman, M. T., Foster, J. D., Nuyujukian, P., Ryu, S. I., et al. (2012). Neural population dynamics during reaching. Nature, 487, 51-56. https://doi.org/10.1038/nature11129

Duque, J., Greenhouse, I., Labruna, L., \& Ivry, R. B. (2017). Physiological markers of motor inhibition during human behavior. Trends in Neurosciences, 40(4), 219-236. https:// doi.org/10.1016/j.tins.2017.02.006

Duque, J., Labruna, L., Verset, S., Olivier, E., \& Ivry, R. B. (2012) Dissociating the role of prefrontal and premotor cortices in controlling inhibitory mechanisms during motor preparation. The Journal of Neuroscience, 32(3), 806-816. https://doi.org/ 10.1523/JNEUROSCI.4299-12.2012

Elsayed, G. F., \& Cunningham, J. P. (2017). Structure in neural population recordings: An expected byproduct of simpler phenomena? Nature Neuroscience, 20(9), 1310-1318. https:// doi.org/10.1038/nn.4617

Genovesio, A., Brunamonti, E., Giusti, M. A., \& Ferraina, S. (2007) Postsaccadic activities in the posterior parietal portex of primates are influenced by both eye movement vectors and eye position. The Journal of Neuroscience, 27(12), 3268-3273. https://doi.org/10.1523/JNEUROSCI.5415-06.2007

Giamundo, M., Giarrocco, F., Brunamonti, E., Fabbrini, F., Pani, P., \& Ferraina, S. (2019). Neuronal activity in the premotor cortex of monkeys reflects both cue salience and motivation for action generation and inhibition. bioRxiv. https://doi.org/ 10.1101/796417, 796417.

Hanes, D. P., Patterson, W. F., \& Schall, J. D. (1998). Role of frontal eye field in countermanding saccades: Visual, movement and fixation activity. Journal of Neurophysiology, 79, 817-834. https://doi.org/10.1152/jn.1998.79.2.817

Hippolyte, L., Iglesias, K., \& Barisnikov, K. (2009). A new emotional stroop-like task: Application to the down syndrome population. Archives of Clinical Neuropsychology, 24(3), 293-300. https://doi.org/10.1093/arclin/acp036

Johnson, P. B., \& Ferraina, S. (1996). Cortical networks for visual reaching: Intrinsic frontal lobe connectivity. European Journal of Neuroscience, 8(7), 1358-1362. https://doi.org/10.1111/j.14609568.1996.tb01598.x

Kaufman, M. T., Churchland, M. M., Ryu, S. I., \& Shenoy, K. V. (2014). Cortical activity in the null space: Permitting preparation without movement. Nature Neuroscience, 7(3), 440-448. https://doi.org/10.1038/nn.3643

Kaufman, M. T., Churchland, M. M., Santhanam, G., Yu, B. M., Afshar, A., Ryu, S. I., et al. (2010). Roles of monkey premotor neuron classes in movement preparation and execution. Journal of Neurophysiology, 104, 799-810. https://doi.org/ 10.1152/jn.00231.2009

Kaufman, M. T., Seely, J. S., Sussillo, D., Ryu, S. I., Shenoy, K. V., \& Churchland, M. M. (2016). The largest response component in the motor cortex refects movement timing but not movement type. eNeuro, 3(4), 85-116. https://doi.org/10.1523/ ENEURO.0085-16.2016

Lijffijt, M., Kenemans, J. L., Verbaten, M. N., \& van Engeland, H. (2005). A meta-analytic review of stopping performance in attention-deficit/hyperactivity disorder: Deficient inhibitory motor control? Journal of Abnormal Psychology, 114(2), 216-222. https://doi.org/10.1037/0021-843X.114.2.216

Lipszyc, J., \& Schachar, R. (2010). Inhibitory control and psychopathology: A meta-analysis of studies using the stop signal task. Journal of International Neuropsychological Society, 16, 1064-1076. https://doi.org/10.1017/S1355617710000895 
Logan, G. D., \& Cowan, W. B. (1984). On the ability to inhibit thought and action: A theory of an act of control. Psychological Review, 91, 295-327. https://doi.org/10.1037/0033-295X.91.3.295

Majid, D. S., Cai, W., George, J. S., Verbruggen, F., \& Aron, A. R. (2012). Transcranial magnetic stimulation reveals dissociable mechanisms for global versus selective corticomotor suppression underlying the stopping of action. Cerebral Cortex, 22(2), 363-371. https://doi.org/10.1093/cercor/bhr112

Mallet, N., Schmidt, R., Leventhal, D., Chen, F., Amer, N., Boraud, T., et al. (2016). Arkypallidal cells send a stop signal to striatum. Neuron, 89, 308-316. https://doi.org/10.1016/ j.neuron.2015.12.017

Mattia, M., Ferraina, S., \& Del Giudice, P. (2010). Dissociated multiunit activity and local field potentials: A theory inspired analysis of a motor decision task. Neuroimage, 52(3), 812-823. https://doi.org/10.1016/j.neuroimage.2010.01.063

Mattia, M., Pani, P., Mirabella, G., Costa, S., Del Giudice, P., \& Ferraina, S. (2013). Heterogeneous attractor cell assemblies for motor planning in premotor cortex. The Journal of Neuroscience, 33(27), 11155-11168. https://doi.org/10.1523/JNEUROSCI.466412.2013

Mirabella, G., Pani, P., \& Ferraina, S. (2011). Neural correlates of cognitive control of reaching movements in the dorsal premotor cortex of rhesus monkeys. Journal of Neurophysiology, 106, 1454-1466. https://doi.org/10.1152/jn.00995.2010

Ogasawara, T., Nejime, M., Takada, \& M., \& Matsumoto, M. (2018). Primate nigrostriatal dopamine system regulates saccadic response inhibition. Neuron, 100(6), 1513-1526. https://doi.org/ 10.1016/j.neuron.2018.10.025

Oldenburg, I. A., \& Sabatini, B. L. (2015). Antagonistic but not symmetric regulation of primary motor cortex by basal ganglia direct and indirect pathways. Neuron, 86(5), 1174-1181. https://doi.org/10.1016/j.neuron.2015.05.008

Pani, P., Di Bello, F., Brunamonti, E., D'Andrea, V., Papazachariadis, O., \& Ferraina, S. (2014). Alpha- and betaband oscillations subserve different processes in reactive control of limb movements. Frontiers in Behavioral Neuroscience, 8, 383. https://doi.org/10.3389/fnbeh.2014.00383

Pani, P., Giamundo, M., Giarrocco, F., Mione, V., Brunamonti, E., Mattia, M., et al. (2019). Neuronal population dynamics during motor plan cancellation in non-human primates. bioRxiv. https://doi.org/10.1101/774307, 774307.

Pani, P., Giarrocco, F., Giamundo, M., Montanari, R., Brunamonti, \& E., \& Ferraina, S. (2018). Visual salience of the stop signal affects the neuronal dynamics of controlled inhibition. Scientific Reports, 8(1), 14265. https://doi.org/10.1038/ s41598-018-32669-8

Pani, P., Menghini, D., Napolitano, C., Calcagni, M., Armando, K., Vicari, S., et al. (2013). Proactive and reactive control of movement are differently affected in Attention Deficit Hyperactivity Disorder children. Research in Developmental Disabilities, 34(10), 3104-3111. https://doi.org/10.1016/ j.ridd.2013.06.032

Paré, M., \& Hanes, D. P. (2003). Controlled movement processing: Superior colliculus activity associated with countermanded saccades. The Journal of Neuroscience, 23, 6480-6489. https:// doi.org/10.1523/JNEUROSCI.23-16-06480.2003

Parmigiani, S., \& Cattaneo, L. (2018). Stimulation of the dorsal premotor cortex, but not of the supplementary motor area proper, impairs the stop function in a STOP signal task. Neuroscience, 394, 14-22. https://doi.org/10.1016/j.neuroscience.2018.10.005

Pouget, P., Logan, G. D., Palmeri, T. J., Boucher, L., Paré, M., \& Schall, J. D. (2011). Neural basis of adaptive response time adjustment during saccade countermanding. The Journal of Neuroscience, 31(35), 12604-12612. https://doi.org/10.1523/ JNEUROSCI.1868-11.2011

Sánchez-Carmona, A. J., Albert, J., \& Hinojosa, J. A. (2016). Neural and behavioral correlates of selective stopping: Evidence for a different strategy adoption. Neuroimage, 139, 279-293. https:// doi.org/10.1016/j.neuroimage.2016.06.043

Scangos, K. W., \& Stuphorn, V. (2010). Medial frontal cortex motivates but does not control movement initiation in the countermanding task. The Journal of Neuroscience, 30(5), 1968-1982. https://doi.org/10.1523/JNEUROSCI.4509-09.2010

Schmidt, R., \& Berke, J. D. (2017). A pause-then-cancel model of stopping: Evidence from basal ganglia neurophysiology. Philosophical transactions of the Royal Society of London. Series B, Biological sciences, 372(1718), 20160202. https://doi.org/10.1098/ rstb.2016.0202

Schmidt, R., Leventhal, D. K., Mallet, N., Chen, F., \& Berke, J. D. (2013). Canceling actions involves a race between basal ganglia pathways. Nature Neuroscience, 16(8), 1118-1124. https://doi.org/10.1038/nn.3456

Sebastian, A., Jung, P., Neuhoff, J., Wibral, M., Fox, P. T., Lieb, K., et al. (2016). Dissociable attentional and inhibitory networks of dorsal and ventral areas of the right inferior frontal cortex: A combined task-specific and coordinate-based meta-analytic fMRI study. Brain Structure \& Function, 221(3), 1635-1651. https://doi.org/10.1007/s00429-015-0994-y

Sebastian, A., Rössler, K., Wibral, M., Mobascher, A., Lieb, K., Jung, P., et al. (2017). Neural architecture of selective stopping strategies: Distinct brain activity patterns are associated with attentional capture but not with outright stopping. The Journal of Neuroscience, 37(40), 9785-9794. https://doi.org/10.1523/ JNEUROSCI.1476-17.2017

Sharp, D. J., Bonnelle, V., De Boissezon, X., Beckmann, C. F., James, S. G., Patel, M. C., et al. (2010). Distinct frontal systems for response inhibition, attentional capture, and error processing. Proceedings of the National Academy of Sciences USA, 107(13), 6106-6111. https://doi.org/10.1073/pnas.1000175107

Shenoy, K. V., Sahani, M., \& Churchland, M. M. (2013). Cortical control of arm movements: A dynamical systems perspective. Annual Review of Neuroscience, 36, 337-359. https://doi.org/ 10.1146/annurev-neuro-062111-150509

Thura, D., \& Cisek, P. (2014). Deliberation and commitment in the premotor and primary motor cortex during dynamic decision making. Neuron, 81(6), 1401-1416. https://doi.org/10.1016/ j.neuron.2014.01.031

Verbruggen, F., Aron, A. R., Band, G. P., Beste, C., Bissett, P. G., Brockett, A. T., et al. (2019). A consensus guide to capturing the ability to inhibit actions and impulsive behaviors in the stopsignal task. eLife, 8, Article e46323. https://doi.org/10.7554/ eLife. 46323

Wagner, J., Wessel, J. R., Ghahremani, A., \& Aron, A. R. (2018). Establishing a right frontal beta signature for stopping action in scalp EEG: Implications for testing inhibitory control in other task contexts. Journal of Cognitive Neuroscience, 30(1), 107-118. https://doi.org/10.1162/jocn_a_01183

Wessel, J. R., \& Aron, A. R. (2017). On the globality of motor suppression: Unexpected events and their influence on behavior and cognition. Neuron, 93(2), 259-328. https://doi.org/ 10.1016/j.neuron.2016.12.013

Williams, B. R., Ponesse, J. S., Schachar, R. J., Logan, G. D., \& Tannock, R. (1999). Development of inhibitory control across the life span. Developmental Psychology, 35, 205-213. https:// doi.org/10.1037/0012-1649.35.1.205

Xu, K. Z., Anderson, B. A., Emeric, E. E., Sali, A. W., Stuphorn, V., Yantis, S., et al. (2017). Neural basis of cognitive control over movement inhibition: Human fMRI and primate electrophysiology evidence. Neuron, 96(6), 1447-1458. https:// doi.org/10.1016/j.neuron.2017.11.010. e6.

Xu, K. Z., Mayse, J. D., \& Courtney, S. M. (2018). Evidence for selective adjustments of inhibitory control in a variant of the stop signal task. Quarterly Journal of Experimental Psychology, 72(4), 818-831. https://doi.org/10.1177/1747021818768721 